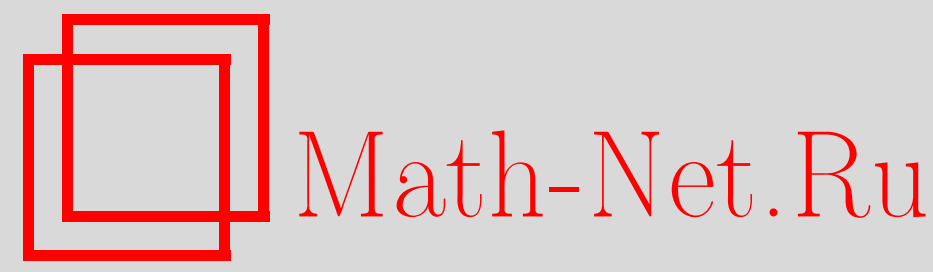

В. М. Климкин, М. Г. Свистула, Свойство Дарбу неаддитивной функции множества, Матем. сб., 2001, том 192, номер 7, 41-50

DOI: https://doi.org/10.4213/sm578

Использование Общероссийского математического портала Math-Net.Ru подразумевает, что вы прочитали и согласны с пользовательским соглашением

http://www . mathnet.ru/rus/agreement

Параметры загрузки:

IP: 52.23 .180 .231

26 апреля 2023 г., $13: 27: 42$ 
УДК 519.53

\author{
В.М. Климкин, М.Г. Свистула
}

\title{
Свойство Дарбу неаддитивной функции множества
}

\author{
Известно, что образом конечной положительной неатомической меры на $\sigma$-ал- \\ гебре является отрезок. В настоящей работе доказано, что это утверждение \\ остается справедливьм для широкого класса неаддитивных функций множества \\ на $F$-алгебре при замене неатомичности свойством Сакса. \\ Библиограбия: 20 названий.
}

К классическим результатам теории меры относится

ТЕОРема 1. Множество значений конечной положительной неатомической меры $\mu$, заданной на б-алгебре $\Sigma$ подмножсеств множсества $T$, есть отрезок $[0, \mu(T)]$.

П. Халмош $[1 ; \S 41$, упражнение 2$]$ отмечает, что эта теорема следует из результатов метрической геометрии, если на $\Sigma$ задать с помощью меры $\mu$ соответствующую метрику. Доказательство теоремы 1 методами теории меры представлено Н. Динкуляну $[2 ; \S 2$, предложение 7].

Теорема 1 многократно передоказывалась и обобщалась различными авторами. $\mathrm{K}$ наиболее значительным результатам можно отнести теорему А.А. Ляпунова о выпуклости и компактности образа $\mathbb{R}^{n}$-значной неатомической меры [3], передоказанную позже методами теории меры П. Халмошем [4] и методами функционального анализа Й. Линденштрауссом [5].

Для конечноаддитивных функций множества со свойством Сакса (см. определение 2 ) на $\sigma$-алгебре аналог теоремы 1 доказал Р. Скоззафава [6], хотя вопрос о связи неатомичности, свойства Сакса и свойства Дарбу (см. определение 1) исследовался еще в [7]. Д. Магарам [8] приводит версию теоремы 1 на $F$-алгебре. В. Олейчеком [9] получены результаты для разложимых мер. Интересный результат о выпуклости образа конечноаддитивной $\mathbb{R}^{n}$-значной меры принадлежит Т. Ф. Армстронгу и К. Прикри [10].

Обобщение теоремы 1 для широкого класса неаддитивных функций множества было получено И. Добраковым [11].

Аналоги теоремы 1 для мер со значениями в группе приведены в работах К. Мусиала [12], Д. Ландерса [13], К. Константинеску [14], А. Мартеллотти [15] и других авторов.

В настоящей статье приводятся некоторые аналоги теоремы 1 для широких классов скалярных функций множества, не обязательно аддитивных. Эти результаты можно использовать, например, в теории неатомических игр, где наибольший интерес представляют функции множества, определенные с помощью неатомических мер (см. [16]). Среди них важнейшими являются, вообше говоря, неаддитивные функции множества вида $f \circ \mu$, где $\mu$ - неатомическая вероятностная мера на $\sigma$-алгебре $\Sigma$ подмножеств множества $T, f$ - функция ограниченной вариации на отрезке $[0,1]$. 
С другой стороны, в связи с потребностями теоретической физики все больший интерес вызывают функции множества с неклассическими областями определения. Так, нам удалось получить некоторые результаты для функции, определенной на $F$-алгебре множеств (см. теорему 2 ).

Приведем ряд используемых в статье обозначений и определений.

Пусть $T$ - некоторое множество, $\Sigma$ - некоторая алгебра подмножеств множества $T$.

Обозначим $E \cap \Sigma=\{A \in \Sigma: A \subset E\}$, где $E \in \Sigma$.

Последовательность попарно непересекающихся множеств $\left\{E_{n}\right\} \subset \Sigma$ будем называть спектром.

Убывающую последовательность множеств $\left\{E_{n}\right\} \subset \Sigma$ с пустьм пересечением будем называть локализатором.

Следуя [17], алгебру множеств $\Sigma$ будем называть $F$-алгеброй, если для любой пары последовательностей из $\Sigma$ (возрастающей $\left\{A_{n}\right\}$ и убывающей $\left\{B_{n}\right\}$, где $\left.A_{n} \subset B_{n}\right)$ существует такое множество $C \in \Sigma$, что $A_{n} \subset C \subset B_{n}$ для всех $n \in \mathbb{N}$.

Очевидно, $\sigma$-алгебра множеств является $F$-алгеброй. Обратное же неверно [17].

В дальнейшем вместо слов “функция множества” будем писать ф.м.

Все рассматриваемые далее функции множества будем предполагать определенньми на алгебре $\Sigma$, принимающими значения из $[0,+\infty)$, равными нулю на пустом множестве.

$\Phi . м . ~ \varphi$ называется исчерпывающей (соответственно непрерывной сверху в нуле), если для любого спектра (соответственно для любого локализатора) $\left\{E_{n}\right\}$ из $\Sigma$ выполняется условие: $\lim _{n \rightarrow \infty} \varphi\left(E_{n}\right)=0$.

$\Phi . м . \varphi$ называется неатомической, если для любого множества $E \in \Sigma$, для которого $\varphi(E)>0$, сушествует такое множество $F \in E \cap \Sigma$, что $\varphi(F) \neq 0$ и $\varphi(E \backslash F) \neq 0$.

$\Phi . м . \varphi$ называется монотонной, если из того, что $A, B \in \Sigma, A \subset B$, следует $\varphi(A) \leqslant \varphi(B)$.

$\Phi . м . \varphi$ будем называть субмерой, если для любого множества $A \in \Sigma$ и для любого числа $\varepsilon>0$ сушествует число $\delta>0$ такое, что если множество $B \in \Sigma$ и $\varphi(B)<\delta$, то

1) $\varphi(A \cup B) \leqslant \varphi(A)+\varepsilon$,

2) $\varphi(A) \leqslant \varphi(A \backslash B)+\varepsilon$.

Монотонные непрерывные сверху в нуле субмеры рассматривались в [11]. Их будем называть субмерами Добракова (см. [11; §1, примеры 1-4]).

$\Phi . м . \varphi$ называем полуаддитивной, если для любого конечного набора $\left\{E_{1}, \ldots, E_{n}\right\}$ попарно непересекаюшихся множеств из $\Sigma$ выполняется

$$
\varphi\left(\bigcup_{i=1}^{n} E_{i}\right) \leqslant \sum_{i=1}^{n} \varphi\left(E_{i}\right) .
$$

Если же вместо неравенства справедливо равенство, то ф.м. $\varphi$ называют $а д д и-$ тивной.

Говорят, что $\varphi-м е р а$, если она счетноаддитивна, т.е. для любого спектра $\left\{E_{n}\right\}$ из $\Sigma$ такого, что $\bigcup_{n=1}^{\infty} E_{n} \in \Sigma$, выполняется

$$
\varphi\left(\bigcup_{n=1}^{\infty} E_{n}\right)=\sum_{n=1}^{\infty} \varphi\left(E_{n}\right) .
$$

Очевидно, монотонная полуаддитивная ф.м., в частности аддитивная ф.м., является субмерой; а мера - субмерой Добракова. 


\section{§1. Свойство Дарбу неаддитивной функции множества на $F$-алгебре}

ОПРЕДЕЛЕНИЕ 1. Будем говорить, что ф.м. $\varphi$ обладает свойством Дарбу (на $\Sigma$ ), если для любого множества $E \in \Sigma$ и для любого числа $0<t<\varphi(E)$ существует такое множество $F \in E \cap \Sigma$, что $\varphi(F)=t$.

ПримеР 1. Пусть $\Sigma-\sigma$-алгебра измеримых по Лебегу подмножеств отрезка $[0,1]$; пусть, далее, $\mu$ - мера Лебега. Через $\Sigma_{0}$ обозначим класс всех тех множеств из $\Sigma$, для которых $\mu(E)=0$ или $\mu(E)=1$.

Для множества $E \in \Sigma_{0}$ положим

$$
\nu_{0}(E)= \begin{cases}0, & \text { если } \mu(E)=0, \\ 2, & \text { если } \mu(E)=1 .\end{cases}
$$

Очевидно, что $\Sigma_{0}$ - подалгебра алгебры $\Sigma$ и $\nu_{0}$ - аддитивная ф.м. на $\Sigma_{0}$. Продолжим $\nu_{0}$ до аддитивной ф.м. $\nu$ на $\Sigma$ так, чтобы множество значений ф.м. $\nu$ содержалось в замыкании множества значений ф.м. $\nu_{0}[18 ; \S 47]$. Следовательно, аддитивная ф.м. $\nu$ принимает два значения: 0 и 2.

Положим $\varphi=\mu+\nu$. Ясно, что $\varphi$ - неатомическая аддитивная ф.м. на $\sigma$-алгебре $\Sigma$ и не принимает ни одного значения из отрезка $[1,2]$, в то время как наибольшее значение ф.м. $\varphi$ на $\Sigma$ равно 3.

Таким образом, для аддитивной ф.м. на $\sigma$-алгебре классической неатомичности недостаточно для выполнения свойства Дарбу. Ранее на этот факт обратил внимание В. Олейчек [9].

Основная цель $\S 1$ - указать условие, на которое надо заменить условие неатомичности, чтобы ф.м. $\varphi$, вообше говоря не являюшаяся непрерьвной, обладала свойством Дарбу. В теореме 2 доказывается, что таким условием для широкого класса неаддитивных функций множества, а именно для субмер на $F$-алгебре, является свойство Сакса.

ОПРЕДЕЛЕНИЕ 2. Будем говорить, что ф.м. $\varphi$ обладает свойством Сакса (на $\Sigma$ ), если для любого множества $E \in \Sigma$ и для любого числа $\varepsilon>0$ существует такое конечное разложение $\left\{E_{1}, E_{2}, \ldots, E_{n}\right\}$ множества $E$, что

$$
\bigcup_{k=1}^{n} E_{k}=E, \quad E_{k} \in \Sigma, \quad E_{i} \cap E_{j}=\varnothing \text { при } i \neq j, \quad \varphi\left(E_{k}\right)<\varepsilon \quad \text { для всех } k \in \overline{1, n} .
$$

ЗАмечАниЕ 1 . Из теоремы 1 сразу следует, что неатомическая мера на $\sigma$-алгебре обладает свойством Дарбу. Легко заметить, что для меры на $\sigma$-алгебре свойства неатомичности, Сакса и Дарбу равносильны.

Теорема 2. Пусть $\Sigma$-некоторая $F$-алгебра подмножеств множества $T$; пусть, далее, $\varphi$ - субмера на $\Sigma$. Если $\varphi$ обладает свойством Сакса, то $\varphi$ обладает свойством Дарбу.

ДоказАтЕльство. Пусть $E \in \Sigma$ и $0<t<\varphi(E)$. Пусть, далее, $\varepsilon_{n}>0, \varepsilon_{n} \rightarrow 0$ при $n \rightarrow \infty$.

По свойству Сакса сушествует конечный набор попарно не пересекаюшихся множеств $\left\{E_{1}^{1}, E_{2}^{1}, \ldots, E_{n_{1}}^{1}\right\} \subset \Sigma$, для которого

$$
\bigcup_{i=1}^{n_{1}} E_{i}^{1}=E, \quad \varphi\left(E_{i}^{1}\right)<\varepsilon_{1}, \quad i=\overline{1, n_{1}} .
$$


Будем считать, что $n_{1} \geqslant 2$, так как в качестве $E_{1}^{1}$ всегда можем взять пустое множество, поскольку $\varphi(\varnothing)=0$.

Тогда найдется такой номер $k_{1}$, что

$$
1 \leqslant k_{1} \leqslant n_{1}-1, \quad \varphi\left(\bigcup_{i=1}^{k_{1}} E_{i}^{1}\right)<t, \quad \varphi\left(\bigcup_{i=1}^{k_{1}+1} E_{i}^{1}\right) \geqslant t .
$$

Положим

$$
A_{1}=\bigcup_{i=1}^{k_{1}} E_{i}^{1}, \quad B_{1}=\bigcup_{i=1}^{k_{1}+1} E_{i}^{1}
$$

Итак,

$$
A_{1} \subset B_{1}, \quad \varphi\left(A_{1}\right)<t, \quad \varphi\left(B_{1}\right) \geqslant t, \quad \varphi\left(B_{1} \backslash A_{1}\right)<\varepsilon_{1} .
$$

Пусть теперь построены множества $A_{1}, \ldots, A_{m-1}, B_{1}, \ldots, B_{m-1}$ из $\Sigma$ такие, что $A_{1} \subset \cdots \subset A_{m-1} \subset B_{m-1} \subset \cdots \subset B_{1}$,

$$
\varphi\left(A_{i}\right)<t, \quad \varphi\left(B_{i}\right) \geqslant t, \quad \varphi\left(B_{i} \backslash A_{i}\right)<\varepsilon_{i}, \quad i=\overline{1, m-1} .
$$

Рассмотрим множество $B_{m-1} \backslash A_{m-1}$. По свойству Сакса сушествует конечный набор попарно не пересекающихся множеств $\left\{E_{1}^{m}, \ldots, E_{n_{m}}^{m}\right\} \subset \Sigma$, для которого

$$
\bigcup_{i=1}^{n_{m}} E_{i}^{m}=B_{m-1} \backslash A_{m-1}, \quad \varphi\left(E_{i}^{m}\right)<\varepsilon_{m}, \quad i=\overline{1, n_{m}} .
$$

Найдется такой номер $k_{m}$, что $1 \leqslant k_{m} \leqslant n_{m}-1$,

$$
\varphi\left(A_{m-1} \cup \bigcup_{i=1}^{k_{m}} E_{i}^{m}\right)<t, \quad \varphi\left(A_{m-1} \cup \bigcup_{i=1}^{k_{m}+1} E_{i}^{m}\right) \geqslant t .
$$

Положим

$$
A_{m}=A_{m-1} \cup \bigcup_{i=1}^{k_{m}} E_{i}^{m}, \quad B_{m}=A_{m} \cup E_{k_{m}+1}^{m} .
$$

Продолжив процесс до бесконечности, получим последовательности множеств $\left\{A_{n}\right\}$ и $\left\{B_{n}\right\}$ из $\Sigma$, для которых

$$
\begin{gathered}
A_{1} \subset \cdots \subset A_{n} \subset \cdots \subset B_{n} \subset \cdots \subset B_{1}, \\
\varphi\left(A_{n}\right)<t, \quad \varphi\left(B_{n}\right) \geqslant t, \quad \varphi\left(B_{n} \backslash A_{n}\right)<\varepsilon_{n}, \quad n \in \mathbb{N} .
\end{gathered}
$$

По определению $F$-алгебры сушествует множество $C \in \Sigma$ такое, что $A_{n} \subset C \subset$ $B_{n}$ для всех $n \in \mathbb{N}$.

Покажем, что $\varphi(C)=t$. Предположим, что $\varphi(C)<t$. Возьмем $\varepsilon=\frac{1}{2}[t-\varphi(C)]$. Для множества $C$ и числа $\varepsilon$ найдем $\delta>0$ такое, что если $B \in \Sigma$ и $\varphi(B)<\delta$, то $\varphi(C \cup B) \leqslant \varphi(C)+\varepsilon$.

Очевидно, существует $n_{0} \in \mathbb{N}$ такое, что $\varepsilon_{n_{0}}<\delta$. Тогда

$$
\varphi\left(B_{n_{0}}\right)=\varphi\left(C \cup\left(B_{n_{0}} \backslash A_{n_{0}}\right)\right) \leqslant \varphi(C)+\varepsilon<t .
$$

Это противоречит тому, что $\varphi\left(B_{n}\right) \geqslant t$ для всех $n \in \mathbb{N}$. Значит, $\varphi(C) \geqslant t$.

Аналогично показывается, что $\varphi(C) \leqslant t$.

Итак, $\varphi(C)=t$. Искомое множество построено. 
СлеДСТВИЕ 1. Для конечноаддитивной функции множества на F-алгебре свойства Сакса и Дарбу равносильны.

ЗАмечАниЕ 2. Как показывает пример 2 , теорема 2, вообше говоря, необратима.

ПримеР 2. Пусть $\Sigma-\sigma$-алгебра измеримых по Лебегу подмножеств вещественной прямой, $\lambda$ - мера Лебега. Для любого $E \in \Sigma$ положим $\varphi(E)=\lambda(E)$, если $\lambda(E) \leqslant 1 ; \varphi(E)=1$, если $\lambda(E)>1$. Как легко заметить, ф.м. $\varphi$ монотонна, полуаддитивна (следовательно, является субмерой), обладает свойством Дарбу и не обладает свойством Сакса.

ЗАмЕчАниЕ 3. Как показывает пример 3 , в теореме 2 предположение о том, что класс множеств $\Sigma$ является $F$-алгеброй, существенно.

ПримеР 3. Рассмотрим полуалгебру промежутков $\langle a, b\rangle$ с рациональньми концами, содержашихся в отрезке $[0,1]$. Пусть $\Sigma$ - алгебра, порожденная этой полуалгеброй, т.е. алгебра подмножеств отрезка $[0,1]$, которые можно представить как объединение конечного числа попарно не пересекающихся промежутков с рациональньми концами. Легко показать, что алгебра $\Sigma$ не является $F$-алгеброй.

Пусть $\lambda$-мера Лебега на $\Sigma$. Очевидно, $\lambda$ обладает свойством Сакса и образом $\lambda$ является множество рациональных чисел отрезка $[0,1]$.

\section{§2. Свойство Сакса непрерывной функции множества}

ТЕОРема 3. Пусть $\Sigma$-некоторая $\sigma$-алгебра подмножеств множества $T$; пусть, далее, $\varphi$ - некоторая функиия мнозсества на $\Sigma$. Если

1) ф.м. $\varphi$ - исчерпьвающая,

2) ф.м. $\varphi$ - непрерывная сверху в нуле,

то для любого множества $E \in \Sigma$ и для любого числа $\varepsilon>0$ существует такое конечное разложение $\left\{E_{1}, E_{2}, \ldots, E_{m}\right\}$ множества $E$, что

$$
E=\bigcup_{i=1}^{m} E_{i}, \quad E_{i} \in \Sigma, \quad E_{i} \cap E_{j}=\varnothing \quad \text { npu } \quad i \neq j
$$

и каждое множество $E_{i}$ есть либо атом ф.м. $\varphi$, для которого $\varphi\left(E_{i}\right)>0$, либо $\varphi\left(E_{i}\right) \leqslant \varepsilon, i=\overline{1, m}$.

ДоказАтельство. Пусть $\varepsilon>0, E \in \Sigma$. Так как ф.м. $\varphi$ исчерпывающая, то существует разве лишш конечное число попарно непересекающихся атомов $\left\{E_{1}, \ldots, E_{p}\right\} \subset E \cap \Sigma$, для которых $\varphi\left(E_{k}\right)>\varepsilon, k=\overline{1, p}$.

Положим $C=E \backslash \bigcup_{k=1}^{p} E_{k}$. Если $\varphi(C) \leqslant \varepsilon$, то теорема доказана.

Рассмотрим случай, когда $\varphi(C)>\varepsilon$.

Положим

$$
a_{1}=\sup \{\varphi(B), B \in C \cap \Sigma, \varphi(B) \leqslant \varepsilon\} .
$$

Заметим, что фигурируюшее в последней формуле семейство множеств содержит пустое множество, так как $\varphi(\varnothing)=0$.

Ясно, что сушествует такое множество $F_{1} \in C \cap \Sigma$, для которого

$$
\frac{1}{2} a_{1} \leqslant \varphi\left(F_{1}\right) \leqslant \varepsilon .
$$


Если $\varphi\left(C \backslash F_{1}\right) \leqslant \varepsilon$, то теорема доказана.

Иначе положим

$$
a_{2}=\sup \left\{\varphi(B), B \in\left(C \backslash F_{1}\right) \cap \Sigma, \varphi(B) \leqslant \varepsilon\right\} .
$$

Тогда сушествует такое множество $F_{2} \in\left(C \backslash F_{1}\right) \cap \Sigma$, для которого

$$
\frac{1}{2} a_{2} \leqslant \varphi\left(F_{2}\right) \leqslant \varepsilon .
$$

Если $\varphi\left(C \backslash\left(F_{1} \cup F_{2}\right)\right) \leqslant \varepsilon$, то теорема доказана; иначе сделаем следующий шаг.

Если процесс продолжается бесконечно, то будут построены невозрастающая последовательность чисел $\left\{a_{k}\right\}$ и спектр $\left\{F_{k}\right\} \subset C \cap \Sigma$ такие, что

$$
0 \leqslant \frac{1}{2} a_{k} \leqslant \varphi\left(F_{k}\right) \leqslant \varepsilon, \quad k \in \mathbb{N} .
$$

Так как $\varphi\left(F_{k}\right) \rightarrow 0$, то $a_{k} \rightarrow 0$ при $k \rightarrow \infty$.

Положим $F=C \backslash \bigcup_{k=1}^{\infty} F_{k}$. Покажем, что $\varphi(F)=0$.

Предположим, что $\varphi(\bar{F})>0$. Если $F$ содержит атомы, то обозначим через $L$ один из атомов; получим, что $L \in F \cap \Sigma$ и $0<\varphi(L) \leqslant \varepsilon$. Если же $F$ не содержит атомов, то в силу исчерпываемости ф.м. $\varphi$ легко доказать сушествование такого множества $L \in F \cap \Sigma$, для которого $0<\varphi(L) \leqslant \varepsilon$.

Тогда по построению $\varphi(L) \leqslant a_{k}$ для любого номера $k$. Переходя к пределу при $k \rightarrow \infty$, получаем, что $\varphi(L)=0$; это противоречит неравенству $\varphi(L)>0$. Значит, $\varphi(F)=0$.

Так как ф.м. $\varphi$ непрерывна сверху в нуле, то существует такой номер $k_{0}$, что

$$
\varphi\left(\bigcup_{k=k_{0}+1}^{\infty} F_{k}\right) \leqslant \varepsilon .
$$

Положим

$$
A=\bigcup_{k=k_{0}+1}^{\infty} F_{k} .
$$

Тогда множества $\left\{E_{1}, E_{2}, \ldots, E_{p}, F_{1}, \ldots, F_{k_{0}}, A, F\right\}$ дают искомое разбиение множества $E$.

ЗАмечАниЕ 4 . В случае, когда ф.м. $\varphi$ - мера, заданная на $\sigma$-алгебре $\Sigma$, из теоремы 3 вытекает известная теорема Сакса о разложении [19].

СлЕДСТВИЕ 2. Пусть $\Sigma-\sigma$-аләебра подмножсеств множества T; пусть, далее, $\varphi$ - неатомическая функиия мноэества на $\Sigma$. Если

1) ф.м. $\varphi-$ исчерпьвающая,

2) ф.м. $\varphi$ непрерывна сверху в нуле, то ф.м. $\varphi$ обладает свойством Сакса.

СЛЕДСТВИЕ 3. На б-алгебре для субмеры Добракова свойства неатомичности, Сакса и Дарбу равносильны.

ДокАзАтЕльство. Пусть $\varphi$ - субмера Добракова на $\sigma$-алгебре $\Sigma$. Так как она монотонна и непрерывна сверху в нуле, то для любого спектра $\left\{E_{n}\right\} \subset \Sigma$ имеем $0 \leqslant \varphi\left(E_{n}\right) \leqslant \varphi\left(\bigcup_{i=n}^{\infty} E_{i}\right)$, где $\varphi\left(\bigcup_{i=n}^{\infty} E_{i}\right) \rightarrow 0$ при $n \rightarrow \infty$. Значит, $\varphi\left(E_{n}\right) \rightarrow 0$ при $n \rightarrow \infty$, т.е. ф.м. $\varphi$ - исчерпьвающая.

Тогда в силу следствия 2 неатомичность влечет свойство Сакса.

Свойство Сакса влечет свойство Дарбу по теореме 2.

Очевидно, из свойства Дарбу следует неатомичность. 


\section{§ 3. Свойство Дарбу и разложение функции множества}

ОПРЕДЕЛЕНИЕ 3. Будем говорить, что ф.м. $\varphi$ имеет $\mathscr{D}$-разложение, если существуют такие монотонные ф.м. $\lambda$ и $\mu$, что выполняются условия:

1) $\varphi(E)=\lambda(E)+\mu(E), E \in \Sigma$;

2) для любого множества $E \in \Sigma$ и для любого числа $\varepsilon>0$ существует такое множество $B \in E \cap \Sigma$, что $\lambda(B)=\lambda(E)$ и $\mu(B)<\varepsilon$.

Пример 4. Пусть $\varphi$-аддитивная ф.м., заданная на $\sigma$-алгебре $\Sigma$; пусть, далее, $\lambda$ и $\mu$ есть разложение ф.м. $\varphi$ в смысле Хьюитта-Иосиды [20], где $\mu$-счетноаддитивная и $\lambda$ - чисто аддитивная составляющие ф.м. $\varphi$. Тогда $\lambda$ и $\mu$ дают $\mathscr{D}$-разложение ф.м. $\varphi$ на $\Sigma$.

Теорема 4. Пусть $\varphi-$ аддитивная ф.м. на F-алгебре $\Sigma$. Если выполняются условия

1) ф.м. $\varphi$ имеет D-разложение $\lambda u \mu$,

2) ф.м. $\lambda$ обладает свойством Дарбу,

3) ф.м. $\lambda$ полуаддитивна,

то для любого множества $E \in \Sigma$ и для любого числа $0 \leqslant t \leqslant \lambda(E)$ существует такое мнохество $A \in E \cap \Sigma$, что $\varphi(A)=t$.

ДокАЗАТЕльство. Возьмем $E \in \Sigma$. Пусть $0<t \leqslant \lambda(E)$. Положим

$$
\varepsilon_{1}=\min (1 ; t)
$$

Так как ф.м. $\varphi$ имеет $\mathscr{D}$-разложение $\lambda$ и $\mu$, то для множества $E$ и числа $\varepsilon_{1}$ существует такое множество $C_{1} \in E \cap \Sigma$, что

$$
\lambda(E)=\lambda\left(C_{1}\right) \quad \text { и } \mu\left(C_{1}\right)<\varepsilon_{1}
$$

Так как ф.м. $\lambda$ обладает свойством Дарбу, то существует такое множество $A_{1} \in$ $C_{1} \cap \Sigma$, что

$$
\lambda\left(A_{1}\right)=t
$$

Очевидно, в силу монотонности ф.м. $\mu$

$$
t=\lambda\left(A_{1}\right) \leqslant \lambda\left(A_{1}\right)+\mu\left(A_{1}\right) \leqslant \lambda\left(A_{1}\right)+\mu\left(C_{1}\right)<t+\varepsilon_{1} .
$$

Таким образом,

$$
t \leqslant \varphi\left(A_{1}\right)<t+\varepsilon_{1}
$$

Из (1) имеем, что $0 \leqslant t-\varepsilon_{1}<\lambda\left(A_{1}\right)$.

Так как ф.м. $\lambda$ обладает свойством Дарбу, то существует такое множество $B_{1} \in$ $A_{1} \cap \Sigma$, что

$$
\lambda\left(B_{1}\right)=t-\varepsilon_{1}
$$

Отсюда в силу монотонности ф.м. $\mu$ следует

$$
t-\varepsilon_{1}=\lambda\left(B_{1}\right) \leqslant \lambda\left(B_{1}\right)+\mu\left(B_{1}\right) \leqslant \lambda\left(B_{1}\right)+\mu\left(C_{1}\right)<t .
$$

Таким образом,

$$
t-\varepsilon_{1} \leqslant \varphi\left(B_{1}\right)<t
$$


Отсюда в силу $(3),(4)$ и полуаддитивности ф.м. $\lambda$ получаем

$$
0<t-\varphi\left(B_{1}\right) \leqslant \varepsilon_{1}=t-\lambda\left(B_{1}\right)=\lambda\left(A_{1}\right)-\lambda\left(B_{1}\right) \leqslant \lambda\left(A_{1} \backslash B_{1}\right) .
$$

Таким образом, сушествуют число $0<\varepsilon_{1} \leqslant 1$, множества $A_{1}, B_{1} \in E \cap \Sigma$ такие, что в силу (5), (4) и (2) выполняется

$$
\begin{gathered}
B_{1} \subset A_{1} \\
0<t-\varphi\left(B_{1}\right) \leqslant \lambda\left(A_{1} \backslash B_{1}\right) \\
0 \leqslant t-\varepsilon_{1} \leqslant \varphi\left(B_{1}\right)<t \leqslant \varphi\left(A_{1}\right)<t+\varepsilon_{1} .
\end{gathered}
$$

Пусть теперь построены число $0<\varepsilon_{n} \leqslant 1 / n$, множества $A_{n}$ и $B_{n}$ из $E \cap \Sigma$ такие, что

$$
\begin{gathered}
B_{n} \subset A_{n}, \\
0<t-\varphi\left(B_{n}\right) \leqslant \lambda\left(A_{n} \backslash B_{n}\right), \\
0 \leqslant t-\varepsilon_{n} \leqslant \varphi\left(B_{n}\right)<t \leqslant \varphi\left(A_{n}\right)<t+\varepsilon_{n} .
\end{gathered}
$$

Положим $\varepsilon_{n+1}=\min \left(1 /(n+1) ; t-\varphi\left(B_{n}\right)\right)$ и найдем в силу $\mathscr{D}$-разложения такое множество

$$
C_{n+1} \in\left(A_{n} \backslash B_{n}\right) \cap \Sigma
$$

чTо

$$
\lambda\left(C_{n+1}\right)=\lambda\left(A_{n} \backslash B_{n}\right) \text { и } \mu\left(C_{n+1}\right)<\varepsilon_{n+1} .
$$

Так как ф.м. $\lambda$ обладает свойством Дарбу, то сушествует такое множество $A_{n+1}^{\prime} \in C_{n+1} \cap \Sigma$, что

$$
\lambda\left(A_{n+1}^{\prime}\right)=t-\varphi\left(B_{n}\right) .
$$

Положим $A_{n+1}=B_{n} \cup A_{n+1}^{\prime}$.

В силу $(7),(6)$ и монотонности ф.м. $\mu$ получим

$$
\begin{aligned}
t & =\varphi\left(B_{n}\right)+\lambda\left(A_{n+1}^{\prime}\right) \leqslant \varphi\left(B_{n}\right)+\varphi\left(A_{n+1}^{\prime}\right) \\
& \leqslant \varphi\left(B_{n}\right)+\lambda\left(A_{n+1}^{\prime}\right)+\mu\left(A_{n+1}^{\prime}\right)<t+\varepsilon_{n+1} .
\end{aligned}
$$

Так как ф.м. $\varphi$ аддитивна, то $\varphi\left(A_{n+1}\right)=\varphi\left(B_{n}\right)+\varphi\left(A_{n+1}^{\prime}\right)$. Отсюда в силу $(8)$ следует, что $t \leqslant \varphi\left(A_{n+1}\right)<t+\varepsilon_{n+1}$.

Аналогично найдем такое множество $B_{n+1}^{\prime} \in A_{n+1}^{\prime} \cap \Sigma$, что $\lambda\left(B_{n+1}^{\prime}\right)=t-$ $\varphi\left(B_{n}\right)-\varepsilon_{n+1}$.

Отсюда в силу монотонности ф.м. $\mu$ получаем

$$
0 \leqslant t-\varepsilon_{n+1} \leqslant \lambda\left(B_{n+1}^{\prime}\right)+\varphi\left(B_{n}\right)+\mu\left(B_{n+1}^{\prime}\right)<t
$$

Положим $B_{n+1}=B_{n} \cup B_{n+1}^{\prime}$.

Тогда из (9), учитывая аддитивность ф.м. $\varphi$, получим $t-\varepsilon_{n+1} \leqslant \varphi\left(B_{n+1}\right)<t$. Отсюда в силу $(7),(8)$ и полуаддитивности ф.м. $\lambda$ получаем

$$
\begin{aligned}
0 & <t-\varphi\left(B_{n+1}\right) \leqslant \varepsilon_{n+1}=\lambda\left(A_{n+1}^{\prime}\right)-\lambda\left(B_{n+1}^{\prime}\right) \\
& \leqslant \lambda\left(A_{n+1}^{\prime} \backslash B_{n+1}^{\prime}\right)=\lambda\left(A_{n+1} \backslash B_{n+1}\right) .
\end{aligned}
$$


Следовательно, существуют число $0<\varepsilon_{n+1} \leqslant 1 /(n+1)$, множества $A_{n+1}$ и $B_{n+1}$ из $E \cap \Sigma$ такие, что

$$
\begin{gathered}
B_{n} \subset B_{n+1} \subset A_{n+1} \subset A_{n}, \\
t-\varphi\left(B_{n+1}\right) \leqslant \lambda\left(A_{n+1} \backslash B_{n+1}\right), \\
0 \leqslant t-\varepsilon_{n+1} \leqslant \varphi\left(B_{n+1}\right)<t \leqslant \varphi\left(A_{n+1}\right)<t+\varepsilon_{n+1} .
\end{gathered}
$$

Продолжив процесс до бесконечности, построим две последовательности множеств $\left\{A_{n}\right\}$ и $\left\{B_{n}\right\}$ из $E \cap \Sigma$ такие, что

$$
\begin{gathered}
B_{n} \subset \cdots \subset B_{n} \subset B_{n+1} \subset \cdots \subset A_{n+1} \subset A_{n} \subset \cdots \subset A_{1}, \\
t-\frac{1}{n} \leqslant \varphi\left(B_{n}\right)<t \leqslant \varphi\left(A_{n}\right)<t+\frac{1}{n}, \quad n \in \mathbb{N} .
\end{gathered}
$$

Так как $\Sigma-F$-алгебра, то существует множество $A \in E \cap \Sigma$ такое, что

$$
B_{n} \subset A \subset A_{n}, \quad n \in \mathbb{N} \text {. }
$$

Отсюда в силу монотонности $\varphi$ следует

$$
t-\frac{1}{n} \leqslant \varphi(A) \leqslant t+\frac{1}{n}, \quad n \in \mathbb{N}
$$

Следовательно, $\varphi(A)=t$. Искомое множество построено.

ТЕОРема 5. Пусть $\varphi-$ аддитивная ф.м. на F-алгебре $\Sigma$ подмножеств мнозества T. Если выполняются условия

1) ф.м. $\varphi$ имеет D-разложение $\lambda u \mu$,

2) ф.м. $\lambda$ обладает свойством Дарбу,

3) ф.м. $\lambda$ полуаддитивна,

4) $\mu(T) \leqslant \lambda(T)$,

то множсеством значений ф.м. $\varphi$ является отрезок $[0, \varphi(T)]$.

ДоКАЗАТЕЛЬСТво. В силу аддитивности ф.м. $\varphi$ достаточно ограничиться случаем $0<t<\frac{1}{2} \varphi(T)$.

Тогда в силу условия 4) из формулировки теоремы имеем $0<t<\lambda(T)$.

Осталось применить теорему 4.

СЛЕДСТВИЕ 4. Если в теореме 5 вместо условия 4) потребовать, чтобъ выполнялось $\mu(E) \leqslant \lambda(E)$ для любого $E \in \Sigma$, то ф.м. $\varphi$ будет обладать свойством Дарбу.

СлЕДСТВИЕ 5. Пусть $\varphi-$ аддитивная ф.м. на б-алгебре $\Sigma$ подмножсеств множества $T$; пусть, далее, $\lambda$ и н есть разложсение Хьюитта-Иосиды, где $\lambda$ - чисто аддитивная и $\mu$-счетноаддитивная ф.м. Если выполнены условия

1) $\lambda$ обладает свойством Дарбу,

2) $\mu(T) \leqslant \lambda(T)$, то множеством значений ф.м. $\varphi$ является отрезок $[0, \varphi(T)]$. 


\section{Список литературы}

1. Халмош П. Теория меры. М.: ИЛ, 1953.

2. Dinculeanu $N$. Vector measures. Berlin: VEB, 1966.

3. Ляпунов A.A. О вполне аддитивных вектор-фуункциях // Изв. АН СССР. Сер. матем. 1940. T. 4. №6. C. 465-478.

4. Halmos P. R. The range of a vector measure // Bull. Amer. Math. Soc. 1948. № 54 . P. 416-421.

5. Lindenstrauss J. A short proof of Liapounoff's convexity theorem // J. Math. Mech. 1966. № 15. P. 971-972.

6. Scozzafava $R$. Completa additivita su opportune successioni di insiemi di una misura di probabilita semplicemente additiva e fortemente non atomica // Boll. Un. Mat. Ital. B(7). 1979. V. 16. № 5. P. 639-648.

7. Rao M. Braskara, Rao K.P. S. Braskara. Charges on Boolean algebras and almost discrete spaces // Mathematika. 1973. № 20. P. 214-223.

8. Maharam D. Finitely additive measures on the integers // Sankhyā Ser. A. 1976. № 38 . P. 44-49.

9. Olejček $V$. Darboux property of finitely additive measure on $\delta$-ring // Math. Slovaca. 1977. V. 27. № 2. P. 195-201.

10. Armstrong T. E., Prikry K. Liapounoff's theorem for nonatomic, finitely-additive, bounded, finite-dimensional, vector-valued measures // Trans. Amer. Math. Soc. 1981. V. 266. № 2. P. 499-514.

11. Dobrakov I. On submeasures. I // Dissertationes Math. 1974. V. 112.

12. Musial K. Absolute continuity and the range of group valued measure // Bull. Acad. Polon. Sci. Ser. Math. Astron. Phys. 1973. V. 21. № 2. P. 105-113.

13. Landers $D$. Connectedness properties of the range of vector and semimeasures // Manuscripta Math. 1973. № 9. P. 105-112.

14. Constantinescu $C$. The range of atomless group valued measures // Comment. Math. Helv. 1976. № 51. P. 207-213.

15. Martellotti $A$. Topological properties of the range of a group-valued finitely additive measure // J. Math. Anal. Appl. 1985. № 110. P. 411-424.

16. Ауман P., Шепли Л. Значения для неатомических игр. М.: Мир, 1977.

17. Seever G. L. Measures on F-spaces // Trans. Amer. Math. Soc. 1968. № 133. P. 267-280.

18. Сикорский Р. Булевы алгебры. М.: Мир, 1969.

19. Saks S. Addition to the note on some functionals // Trans. Amer. Math. Soc. 1933. № 35. P. 967-974.

20. Yosida K., Hewitt E. Finitely additive measures // Trans. Amer. Math. Soc. 1952. № 72. P. $46-66$. 